\title{
Epidemiology of childhood tuberculosis in Switzerland between 1996 and 2011
}

\author{
Gabriela Oesch Nemeth • Johannes Nemeth • \\ Ekkehardt Altpeter • Nicole Ritz
}

Received: 19 August 2013 /Revised: 21 October 2013 / Accepted: 21 October 2013 / Published online: 8 November 2013

(C) Springer-Verlag Berlin Heidelberg 2013

\begin{abstract}
Approximately 9 million cases of tuberculosis (TB) are reported annually and half a million occur in children $<15$ years of age. Globally, TB notifications in children have been neglected for decades although childhood TB may represent a sentinel for ongoing transmission. Data included in this study were collected from the TB database of the Swiss Federal Office of Public Health, which includes culture-confirmed TB and/or cases treated with $\geq 3$ anti-mycobacterial drugs. Data from all children $<15$ years of age reported between 1996 and 2011 were analyzed. A total of 320 cases of TB (166 cultures confirmed, 5 confirmed by nucleic acid amplification, 149 other than definite cases) were reported with an overall incidence rate of 1.6 per 100,000 children (range 1.2-2.2). A total of 154 $(48 \%)$ children were younger than 5 years of age and 141 (44\%) were born in Switzerland. Children below 5 years of age were more likely to be Swiss-born compared to children aged 10 to 14 years ( $74 \%$ versus $26 \%$ ). When analyzing the country of origin, only 55 children $(17 \%)$ were of Swiss origin. Of all children with foreign origin, 117 (47\%) were from a
\end{abstract}

\author{
G. Oesch Nemeth \\ University Children's Hospital, Zürich, Switzerland \\ J. Nemeth \\ Division of Infectious Diseases and Hospital Epidemiology, \\ University Hospital Zürich, University of Zürich, Zurich, \\ Switzerland \\ E. Altpeter \\ Federal Office of Public Health, Bern, Switzerland \\ N. Ritz $(\bowtie)$ \\ Infectious Diseases Unit, University Children's Hospital Basel, the \\ University of Basel, Spitalstrasse 33, 4031 Basel, Switzerland \\ e-mail: nicole.ritz@unibas.ch \\ N. Ritz \\ Department of Paediatrics, The University of Melbourne, Parkville, \\ Australia
}

country within the WHO European Region. In 288 (90 \%) of all notified cases, the site of disease was the lung. Mycobacterial culture was positive in 166 cases $(51.9 \%)$ with $1.8 \%$ multidrug-resistance. The overall incidence of childhood TB disease reported in Switzerland remained stable over a 16-year period with a remarkable high rate of very young patients of foreign origin. Only half of the reported cases were culture confirmed, highlighting the need for better diagnostic tests in childhood TB.

Keywords Tuberculosis $\cdot$ Epidemiology $\cdot$ Migration $\cdot$ Child · MDR-TB · TB

\section{Introduction}

Worldwide, approximately 9 million cases of tuberculosis (TB) are reported annually and it is estimated that half a million occur in children $<15$ years of age [23]. The greatest burden of TB is reported in sub-Saharan Africa and Asia while cases from the World Health Organization (WHO) European region represent approximately $5 \%$ of all TB cases worldwide $[9,23]$. Globally, TB incidence rates are declining. However, international migration influences TB epidemiology resulting in an increase of TB notification rates in some European countries including Malta, Iceland, Cyprus, Sweden and the UK since $2004[12,13]$. In the UK, the majority $(61 \%)$ of TB cases reported in 2004 occurred in foreign-born residents [1].

While in adults, epidemiological data of TB have been collected and reported by the WHO since the 1990s, special consideration and reporting of childhood TB has only been incorporated in the most recent WHO report [13]. Challenges in the diagnosis of TB in children and a high proportion of smear-negative cases have led to underreporting. Consequently, childhood TB was often not prioritised by national control programmes and reliable epidemiological data are scarce [1, 10]. TB generally results from recent transmission from a 
smear-positive contact, mainly household or school contacts [2]. Therefore, analysis of epidemiological data in children is important as it may represents a sentinel for ongoing transmission and it also serves as a measure of the overall TB control [1].

In Switzerland, epidemiological data of TB in children have been collected for more than a decade but were never analyzed separately in detail [5]. The aim of this study was to analyze epidemiological and clinical data of childhood TB in Switzerland and compare results with data from other studies in countries with low TB incidence.

\section{Patients and methods}

Data were collected from the TB database of the Swiss Federal Office of Public Health (FOPH) which included all children with confirmed TB (positive culture) and those with presumed TB treated with three or more anti-mycobacterial drugs. TB notifications in Switzerland are mandatory for both microbiology laboratories and clinicians initiating anti-tuberculous treatment. Microbiology laboratories have to report to the FOPH and the regional health authority (cantonal physician); clinicians have to report to the cantonal physician, who forwards this notification to the FOPH. All notifications are collected in a centralized database, information is linked by personal identifiers and entered as one record per case. Results from all children below 15 years of age reported from 1996 to 2011 were included for this study.

Swiss origin was defined as the country of birth being Switzerland and/or Swiss nationality. In the case of evidence for foreign birth or foreign nationality, the case was defined as foreign origin. If both the country of birth and nationality were unknown, the origin was defined as unknown.

For this study, confirmed TB disease was defined as a positive result from culture or nucleic acid amplification technique (NAAT) of sputum, bronchoalveolar lavage fluid, gastric aspirate, lymph node, bone biopsy, joint fluid, cerebrospinal fluid with a species within the Mycobacterium tuberculosis complex (Mycobacterium tuberculosis, Mycobacterium bovis and Mycobacterium africanum). Major sites of disease were grouped into pulmonary (lung parenchyma, tracheobronchial tree, intrathoracic lymph nodes), pleural, extrathoracic lymph nodes, bones and joints, gastrointestinal, meningitis, and disseminated TB according to the clinician's assessment. All other sites of disease not further specified were grouped into unknown.

\section{Results}

A total of 320 (166 culture confirmed, 5 NAAT confirmed, 149 treated with $\geq 3$ anti-mycobacterial drugs) cases of TB in children below 15 years of age were reported over a 16-year period. The overall mean incidence rate was 1.6 (median 1.7, range $1.2-2.2$ ) per 100,000 children per year, which varied from year to year but without an obvious trend over time (Fig. 1).

The mean age of all children with TB was 5.9 with a standard deviation (SD) of 4.7 years (median 5.0, interquartile range (IQR) 2.0 to 10.0 years) and 165 (52\%) were female (Fig. 2). A total of 154 cases ( $48 \%$ ) were reported in children below 5 years of age.

When analyzing the country of birth, 141 (44\%) children were foreign-born, 141 (44\%) children were Swiss-born, and in 38 cases (12\%), the place of birth was unknown (Fig. 3a). Children below 5 years of age were more likely to be Swissborn compared to children aged 10 to 14 years (67\% versus $15 \%$, respectively). However, when analyzing country of origin, only $55(17 \%)$ children were of Swiss origin, 262 $(82 \%)$ were of foreign origin and in $3(1 \%)$ children origin was unknown. Of all children with foreign origin and known citizienship, 117 (47\%) were from a country within the WHO European Region (Table 1). The mean age of all children with Swiss origin was 4.2 years (median 3 , range $0-13$ ), the mean age of all children with foreign origin was 6.3 years (median 5.5 , range $0-14$ ).

When analyzing the site of infection of all reported cases, pulmonary TB was found in $288(90 \%)$ of the reported TB cases, of which 26 were classified as hilar lymphadenopathy. Of all children with pulmonary TB, 145 (50.3\%) were reported in children younger than 5 years of age. The second most commonly affected site were extra-thoracic lymph nodes with $16(5 \%)$ cases. Other reported sites were the central nervous system (three cases), the pleural space (two cases), bones and joints (two cases), and one case each in the vertebral column,
Fig. 1 Overall tuberculosis incidence rate in children below 15 years of age notified in Switzerland

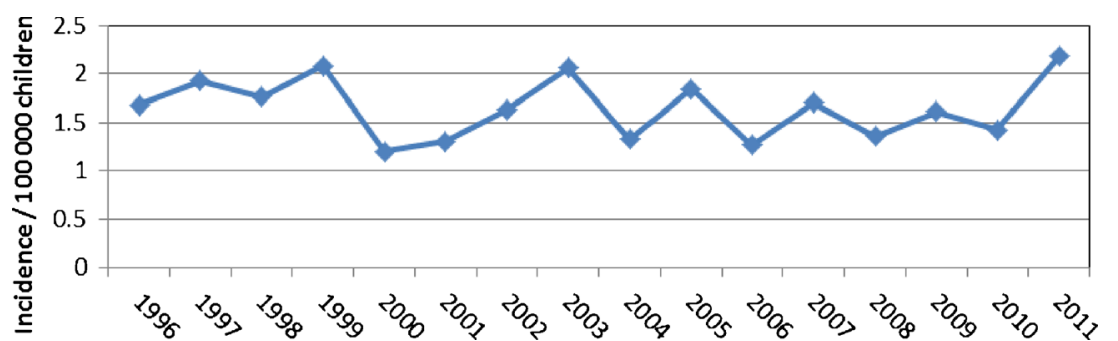




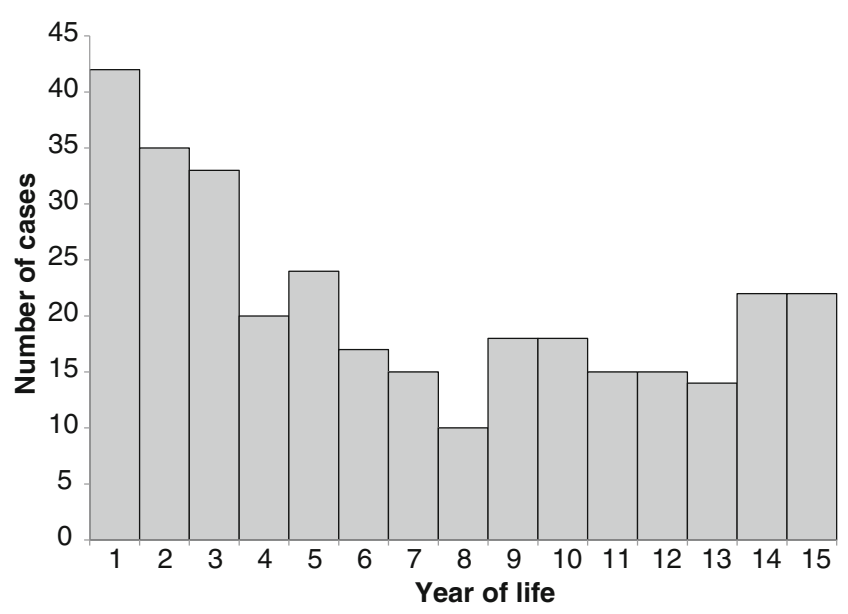

Fig. 2 Age distribution of all children below 15 years of age with tuberculosis reported in Switzerland between 1996 and 2011

gastrointestinal tract and disseminated TB. In six cases, the site of disease was unknown.

Mycobacterial culture was positive in 166 cases (51.9\%); Mycobacterium tuberculosis in 153 cases and Mycobacterium africanum in 3 cases were isolated. In 10 culture-positive cases, the mycobacterial species was not reported. Culture was reported as negative in four cases $(1.3 \%)$, and in 150 cases $(46.9 \%)$, the result of the culture was reported as unknown at the time of notification but remained negative. The mean age of all children with a positive culture was 6.1 (SD 5.0) years (median 5.0 years, IQR 1 to 11). In the age group 0 to 4 years, $51.3 \%$ were culture positive, in the 5 - to 9 year group, $46.2 \%$ and in the 10 - to 14 -year group, $58.0 \%$. When we analyzed the site of infection only in cultureconfirmed cases, 147 of $166(89 \%)$ were classified as pulmonary TB and 4 of $166(5 \%)$ were classified as extra-thoracic lymph node TB.

Monoresistance was reported for isoniazid in nine isolates and for rifampicin and ethambutol in three isolates each. Only three cases $(1.8 \%)$ were reported to be multi-drug-resistant (MDR) TB, defined as resistant to both isoniazid and rifampicin.

\section{Discussion}

Incidence of childhood TB in Switzerland remains low with a rate of 1,6 per 100,000. However, calculation of incidence rates represents approximations as currently Swiss population statistics do not include asylum seekers. Between 1996 and 2011, an estimated 75,000 to 100,000 individuals were living in Switzerland applying for asylum which until 2010 were not included in the population statistics, and therefore, true incidence rates may be lower. Similar low incidence rates have been reported in England and Wales with 4.3 TB cases per 100,000 children per year between 1999 and 2006 [1]. This is in contrast to incidence rates from high-burden countries, such as South Africa, with an estimated TB incidence rate of 237 per 100,000 children [13]. TB notification rates for children in
Fig. 3 Country of birth (a) and country of origin $(\mathbf{b})$ in children aged $0-4$ years $(n=154), 5-$ 8 years $(n=78)$ and $10-14$ years $(n=88)$ notified with tuberculosis in Switzerland
A

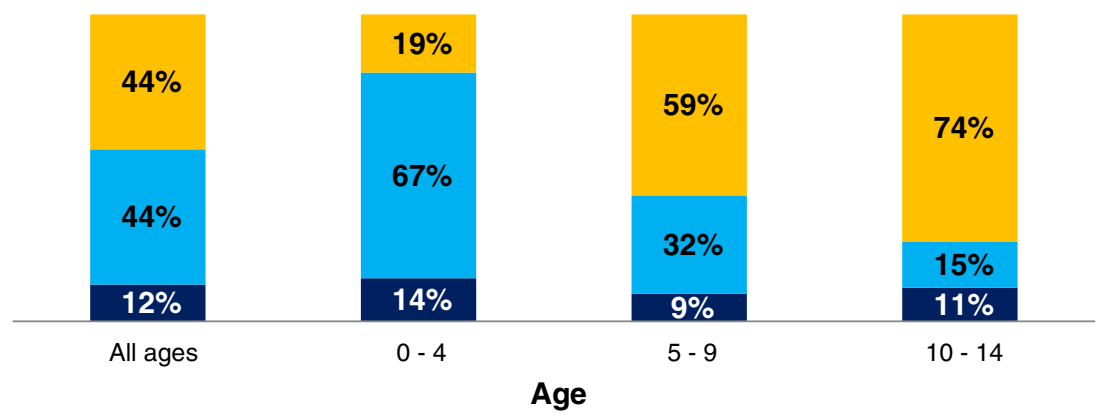

B

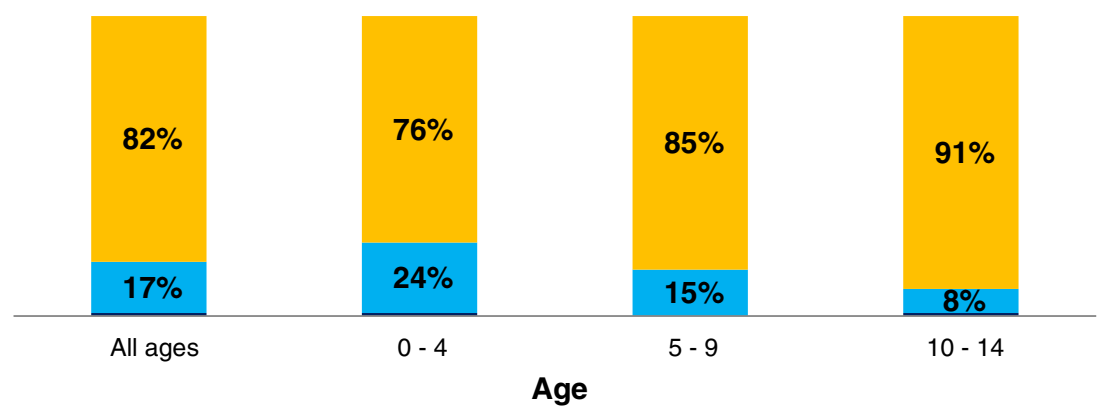


Table 1 Countries of citizenship from the 262 children with foreign origin a Children with foreign origin, who have obtained Swiss citizenship or children with Swiss citizenship who were born abroad

\begin{tabular}{|c|c|c|c|}
\hline Country & No. of patients $(\%)$ & Country & No. of patients $(\%)$ \\
\hline Serbia & $62(23.7)$ & Sudan & $2(0.8)$ \\
\hline Somalia & $30(11.5)$ & Senegal & $2(0.8)$ \\
\hline Portugal & $14(5.3)$ & Papua New Guinea & $1(0.4)$ \\
\hline Switzerland $^{\mathrm{a}}$ & $12(4.6)$ & Sierra Leone & $1(0.4)$ \\
\hline Albania & $12(4.6)$ & Togo & $1(0.4)$ \\
\hline Eritrea & $11(4.2)$ & Guinea-Bissau & $1(0.4)$ \\
\hline Bosnia Herzegovina & $7(2.7)$ & Dominic. Republic & $1(0.4)$ \\
\hline Mazedonia & $7(2.7)$ & Russia & $1(0.4)$ \\
\hline Turkey & $6(2.3)$ & Greece & $1(0.4)$ \\
\hline China and Tibet & $6(2.3)$ & Spain & $1(0.4)$ \\
\hline Angola & $6(2.3)$ & France & $1(0.4)$ \\
\hline Kongo (Brazzaville) & $4(1.5)$ & Ukraine & $1(0.4)$ \\
\hline Sri Lanka & $4(1.5)$ & Iran & $1(0.4)$ \\
\hline Côte d'Ivoire & $4(1.5)$ & Afghanistan & $1(0.4)$ \\
\hline Iraq & $3(1.1)$ & Japan & $1(0.4)$ \\
\hline Pakistan & $3(1.1)$ & Thailand & $1(0.4)$ \\
\hline Croatia & $3(1.1)$ & China (Taiwan) & $1(0.4)$ \\
\hline Kenia & $3(1.1)$ & Armenia & $1(0.4)$ \\
\hline Ruanda & $3(1.1)$ & Georgia & $1(0.4)$ \\
\hline Tschad & $3(1.1)$ & Ethiopia & $1(0.4)$ \\
\hline Romania & $2(0.8)$ & Ghana & $1(0.4)$ \\
\hline Italy & $2(0.8)$ & Mali & $1(0.4)$ \\
\hline India & $2(0.8)$ & Brazil & $1(0.4)$ \\
\hline Cambodia & $2(0.8)$ & Chile & $1(0.4)$ \\
\hline Vietnam & $2(0.8)$ & Ecuador & $1(0.4)$ \\
\hline Cameroun & $2(0.8)$ & Peru & $1(0.4)$ \\
\hline Guinea & $2(0.8)$ & Unknown/stateless & $15(5.7)$ \\
\hline Madagascar & $2(0.8)$ & & \\
\hline
\end{tabular}

Switzerland were lower compared to the overall incidence rates, which were reported to be 7.1 per 100,000 inhabitants in 2009 [4]. Similar observations were reported in other European countries with three to four times higher incidence in all age groups above 14 years of age [17].

In our study, almost half of the notified patients were below 5 years of age. This is in accordance with data from the European Union with approximately $50 \%$ of TB cases in children reported below the age of 5 years [17].

A similar number of children with TB were Swiss-born and foreign-born; however, the proportion of Swiss-born children was higher in the group aged 0 to 4 years. Importantly, many of the Swiss-born children had foreign citizenship, and overall, only one in five children with TB in Switzerland were of Swiss origin. Children with Swiss origin were younger than those with foreign origin and two thirds of all children of Swiss origin with TB were under 5 years of age. Similar trends have been found in other low-TB-incidence countries showing that the largest proportion of children with TB aged below 5 years were nationally born $[1,17,22]$. In our study, a more detailed analysis including the country of birth and country of origin shows that a large proportion of these children categorized as nationally born descend from foreign-born parents. Their parents likely have immigrated recently from a highTB-incidence country which explains the increased risk of transmission and TB disease in their children [16, 22].

Older children with TB notified in Switzerland are more likely to be of foreign origin. Given the low incidence of TB in Switzerland, the most likely explanation is acquisition of TB in their country of origin either before they arrived in Switzerland, during recent travel to a high-TB-incidence country, or from friends and relatives with a recent diagnosis of TB disease [15].

Switzerland has abandoned the universal TB screening and introduced a questionnaire-based case finding of all immigrants in 2006, and therefore, routine TST/IGRAs and chest X-rays at first entry are no longer done [18]. Unfortunately, the current database does allow analysis on the interval between arrival in Switzerland and TB notification.

Most patients of foreign origin were from the former Yugoslavia or from Somalia, the latter with estimated TB 
incidence rates of 150 per 100,000 inhabitants [23]. Although immigration to Switzerland increased during the study period with over $20 \%$ foreign residents in 2011, incidence rates of childhood TB remained stable during the study period [6]. One possible explanation is that there has been a slight shift towards an origin from a low-TB-incidence country in people immigrating to Switzerland [6]. However, incidence rates in adults have increased between 2007 and 2011 [4, 5]. It is therefore possible that increased awareness, early treatment of adults/parents and meticulous contact tracing and preventative therapy with isoniazid have prevented the transmission of TB from adults to children and progression of TB infection to disease in children. Unfortunately, epidemiological data on children with latent TB is not systematically collected, and therefore, it remains elusive if more children have been treated for latent TB in the same time period [20].

In our study, TB was culture-confirmed in over half of all reported cases. This proportion of culture-confirmed cases is considerably higher compared to that reported across Europe ranging from 15 to $20 \%[1,17]$. However, a Swedish study including 147 children below 18 years of age showed comparable results [12]. The higher rate of microbiological confirmation in some countries can be explained by underreporting of culture-negative TB cases in other countries. It may, however, reflect the willingness of both clinicians and families to collect samples from children with suspected TB for microbiological confirmation combined with an optimal mycobacterial detection rate of well-equipped laboratories in Switzerland. The differences in proportions of culture confirmed cases in children in different countries may also reflect over-reporting, which is rarely discussed. However, until more accurate diagnostic tests for TB are available for children, both underreporting and overreporting will occur [7, 8]. The low incidence of MDR TB is comparable to other pediatric studies and reflects MDR TB rates in adult patients in Switzerland [1, 3, 5, 12]. Nevertheless, MDR TB remains of concern as experience for treatment of these children is limited as a result of few children being diagnosed with MDR TB in Switzerland. In addition, optimal management of children exposed to adults with MDR TB is unclear and guidance on a national and international level is consequently based on expert opinion [11, 19, 21].

As a result of the limited set of variables collected by the FOPH according to European recommendations, several other potentially interesting epidemiological and clinical data could not be investigated [14]. This includes the rates of infection with the Human Immunodeficiency Virus, Bacille CalmetteGuérin (BCG) vaccination and detailed results from NAAT and immuno-diagnostic tests including the tuberculin skin test and/or interferon gamma release assays. We are also unable to examine underreporting or over-reporting as data of TB in children in Switzerland is currently not collected in any other systematic way. However, the Swiss Paediatric Surveillance Unit has recently accepted to include TB into their surveillance system. This will allow more detailed analysis of the epidemiology of children with TB in Switzerland and also enhance analysis of potential underreporting or overreporting in the future.

Acknowledgments NR is supported by a career advancement grant from the University of Basel and the Rozalia foundation.

Conflict of interest None of the authors declare a conflict of interest.

\section{References}

1. Abubakar I, Laundy MT, French CE, Shingadia D (2008) Epidemiology and treatment outcome of childhood tuberculosis in England and Wales: 1999-2006. Arch Dis Child 93:1017-1021

2. Basu Roy R, Sotgiu G, Altet-Gomez N, Tsolia M, Ruga E, Velizarova S, Kampmann B (2012) Identifying predictors of interferon-gamma release assay results in pediatric latent tuberculosis: a protective role of bacillus Calmette-Guerin?: a pTB-NET collaborative study. Am J Respir Crit Care Med 186:378-384

3. Brandli O, Zellweger JP (2011) Tuberculosis in Switzerland. Ther Umsch Rev Therapeutique 68:365-368

4. Bundesamt für Gesundheit (2011) Tuberkulose in der Schweiz 20052009. Bull BAG 10:205-213

5. Bundesamt für Gesundheit (2013) Tuberkulose in der Schweiz 2005 bis 2011. Bull BAG 21:343-346

6. Bundesamt für Statistik (2011) Bevölkerung. http://www.bfs.admin. ch/. Accessed 18 Aug 2013

7. Connell T, Tebruegge M, Ritz N, Curtis N (2009) Interferon-gamma release assays for the diagnosis of tuberculosis. Pediatr Infect Dis J 28:758-759

8. Connell TG, Tebruegge M, Ritz N, Bryant P, Curtis N (2011) The potential danger of a solely interferon-gamma release assay-based approach to testing for latent Mycobacterium tuberculosis infection in children. Thorax 66:263-264, author reply 265

9. European Centre for Disease Prevention and Control, WHO Regional Office for Europe (2013) Tuberculosis surveillance and monitoring in Europe 2013. Stockholm: European Centre for Disease Prevention and Control

10. Graham SM (2011) Treatment of paediatric TB: revised WHO guidelines. Paediatr Respir Rev 12:22-26

11. Lungenliga Schweiz, Bundesamt für Gesundheitswesen (2012) Handbuch Tuberkulose. http://www.tbinfo.ch/uploads/media/ Handbuch_Tuberkulose_2012_de.pdf. Accessed 18 Aug 2013

12. Nejat S, Buxbaum C, Eriksson M, Pergert M, Bennet R (2012) Pediatric tuberculosis in Stockholm: a mirror to the world. Pediatr Infect Dis J 31:224-227

13. Nelson LJ, Wells CD (2004) Global epidemiology of childhood tuberculosis. Int J Tuberc Lung Dis 8:636-647

14. Rieder HL, Watson JM, Raviglione MC, Forssbohm M, Migliori GB, Schwoebel V, Leitch AG, Zellweger JP (1996) Surveillance of tuberculosis in Europe. Working Group of the World Health Organization (WHO) and the European Region of the International Union Against Tuberculosis and Lung Disease (IUATLD) for uniform reporting on tuberculosis cases. Eur Respir J 9:1097-1104

15. Ritz N, Connell TG, Curtis N (2008) To BCG or not to BCG? Preventing travel-associated tuberculosis in children. Vaccine 26: 5905-5910

16. Ruwende JE, Sanchez-Padilla E, Maguire H, Carless J, Mandal S, Shingadia D (2011) Recent trends in tuberculosis in children in London. J Public Health (Oxf) 33:175-181 
17. Sandgren A, Hollo V, Quinten C, Manissero D (2011) Childhood tuberculosis in the European Union/European Economic Area, 2000 to 2009. Euro surveill 16(12):pii=19825

18. Schneeberger Geisler S, Helbling P, Zellweger JP, Altpeter ES (2010) Screening for tuberculosis in asylum seekers: comparison of chest radiography with an interview-based system. Int J Tuberc Lung Dis 14:1388-1394

19. Seddon JA, Godfrey-Faussett P, Hesseling AC, Gie RP, Beyers N, Schaaf HS (2012) Management of children exposed to multidrugresistant Mycobacterium tuberculosis. Lancet Infect Dis 12:469-479

20. Tebruegge M, Salo E, Ritz N, Kampmann B, On Behalf Of The Paediatric Tuberculosis Network European Trialsgroup P (2013)
Inclusion of latent tuberculosis infection as a separate entity into the international classification of diseases. Thorax 68:588

21. The Sentinel Project for Pediatric Drug-Resistant Tuberculosis (2012) Management of drug-resistant tuberculosis in children: a field guide. http://sentinelproject.files.wordpress.com/2012/11/sentinel project_field_guide_2012.pdf. Accessed 20 Oct 2013

22. Winston CA, Menzies HJ (2012) Pediatric and adolescent tuberculosis in the United States, 2008-2010. Pediatrics 130: e1425-e1432

23. World Health Organization (2012) Global tuberculosis report 2012. http://www.who.int/tb/publications/global_report/en/. Accessed 18 Aug 2013 